\title{
Proposta de um instrumento para mensurar a satisfação de clientes de e-commerce
}

\author{
Cristina Giacomel Bacharel em Administração. Universidade Federal da Fronteira \\ Sul (UFFS) - Brasil. cristinagiacomel@yahoo.com \\ Janaína Gularte Cardoso Bacharel e Mestre em Administração. Universidade Federal de \\ Santa Catarina (UFSC) - Brasil. janaina.cardoso@uffs.edu.br \\ Carlos Alberto do Espírito Santo Júnior Mestrando em Administração. Universidade Federal de Santa \\ Catarina (UFSC) - Brasil. carlosalberto.espiritosanto@gmail.com
}

\section{RESUMO}

A satisfação dos clientes tem se caracterizado como um dos principais objetivos das organizações que desejam manter a competitividade e sobreviver à concorrência do mercado. Clientes satisfeitos são mais suscetíveis a tornarem-se leais à empresa, repetirem suas compras e realizarem boca a boca positivo. No entanto, apesar do constante crescimento apresentado pelo segmento do e-commercenos últimos anos, percebe-se que há uma carência de metodologias voltadas à mensuração da satisfação do consumidor on-line. Nesse sentido, este trabalho tem o objetivo de propor um instrumento de mensuração do nível de satisfação de clientes para o segmento de e-commerce. Os modelos SERVQUAL, SERVPERF, Modelo Brasileiro Quali Quanti e os Barômetros Nacionais (Modelo Sueco, Americano e Europeu), que são metodologias encontradas na literatura para mensurar a satisfação dos clientes, foram escolhidos para fundamentar este estudo. 0 instrumento proposto foi validado empiricamente através da aplicação de uma pesquisa Surveyem um dos maiores varejos on-line do Brasil, onde foram obtidas 384 respostas. Além disso, aplicou-se o Alfa de Cronbach com o propósito de verificar a confiabilidade do instrumento, que demonstrou bons índices em quatro das seis dimensões originais do instrumento. Ao final do estudo, foi possível adequar o instrumento proposto para torná-lo ainda mais preciso em relação à avaliação da satisfação dos clientes de e-commerce.

Palavras-chave: Pesquisa de satisfação de clientes. Método de mensuração da satisfação. Varejo eletrônico. Consumidor virtual.

\section{Proposal for an instrument to measure satisfaction of e-commerce clients}

\begin{abstract}
Consumer satisfaction has been one of the main goals for organizations that want to keep competitiveness, surviving to market competitors. Satisfied customers are more prone to be loyal to a company, repeating their shopping and spreading a positive word-of-mouth. However, considering e-commerce steady growth in the past few years, a lack of methodologies able to measure the on-line consumer's satisfaction is perceived. In this context, the present work aims to propose a measurement method to identify the level of satisfaction of e-commerce customers. Methodologies found in literature, as SERVQUAL, SERVPERF, Brazilian Quali Quanti Model and the National Barometers (Swedish, American and European Models), were used to base this study. The proposed method was empirically validated through a survey about one of the greatest Brazilian on-line store, obtaining 384 answers. After that, Cronbach's alpha was applied aiming to verify the instrument reliability, demonstrating good indices to four of the six original instrument dimensions. By the end of study, the model was reformulated to make it more adherent for e-commerce costumer's satisfaction evaluations.
\end{abstract}

Keywords: Customer Satisfaction Survey. Method of Measurement of Satisfaction. E-retail. Virtual Consumer 


\section{INTRODUÇÃO}

Nas últimas décadas, o crescimento da economia brasileira, o aumento do poder de compra do consumidor, a ampliação da oferta de bens e serviço e 0 aumento da participação de empresas estrangeiras em diversos setores econômicos colaboraram para que a concorrência se tornasse cada vez mais acirrada no mercado nacional (LOPES; PEREIRA; VIEIRA, 2009). Assim, os mercados não se encontram apenas mais dinâmicos, mas também mais arriscados, complexos e desordenados (AAKER, 2012).

Neste cenário, o papel do marketing vem ganhando destaque e observa-se uma crescente atenção acadêmica para o estudo da satisfação do cliente, seus antecedentes e consequentes (URDAN; RODRIGUES, 1999) e na compreensão dos fatores que levam o consumidor a repetir suas compras por longos períodos de tempo.

Nesse sentido, a maior ênfase dada à satisfação dos clientes teve como consequência o desenvolvimento de métodos que pudessem ser utilizados para mensurá-la. De acordo com Marchetti e Prado (2004), há vários instrumentos que podem ser utilizados na avaliação da satisfação do consumidor, dos quais se podem destacar os modelos baseados no paradigma da desconformidade, representado pelo SERVQUAL; outros baseados na percepção de performance, metodologia presente na escala SERVPERF; e aqueles modelos baseados em equações estruturais, dentre os quais destacam-se os Barômetros nacionais. Além desses modelos, existem metodologias que também mensuram a satisfação dos clientes através da análise de suas percepções, como o Modelo Brasileiro Quali Quanti.

Ainda sob esse entendimento, a temática da satisfação dos clientes é um tópico que merece atenção dos gestores em qualquer tipo e tamanho de negócio. Com o advento e a popularização da internet, 0 ecommerce vem se tornando um canal de compras que tem, cada vez mais, se popularizado e se consolidado nos últimos anos. No entanto, há uma lacuna entre o comportamento do consumidor on-line e aspectos que influenciam suas compras e geram satisfação ou insatisfação.

Nesse enfoque, Baptista, Silva e Goss (2011) destacam que cresce a busca de um melhor entendimento do comportamento do consumidor na internet e dos processos que fazem com que os clientes se mantenham ou não leais a uma determinada empresa no ambiente virtual.

Diante do exposto, o objetivo geral deste estudo visa a propor um instrumento de mensuração do nível de satisfação de clientes do segmento de e-commerce do tipo Bussiness-to-Consumer (B2C), baseado nas características dos modelos de mensuração da satisfação já existentes.

0 propósito é aquecer as discussões acerca do tema, promovendo reflexões e novas contribuições para essa área que se encontra em constante mutação. As principais contribuições que este artigo traz para o assunto são: resgatar os principais estudos teóricos sobre a satisfação dos clientes; levantar atributos dos consumidores habituais de e-commerce, para identificar o que os motiva a comprar pela internet; propor um instrumento de mensuração da satisfação de clientes de e-commerce e validar empiricamente o modelo, em uma loja de varejo virtual de abrangência nacional.

Este trabalho está estruturado em seis partes. A primeira parte é composta por esta introdução. Na segunda parte, apresenta-se a fundamentação teórica, com os assuntos que permitiram embasar o desenvolvimento deste estudo. Na terceira parte, encontram-se os procedimentos metodológicos adotados para alcançar os objetivos estabelecidos e em seguida, na quarta parte, apresenta-se a análise dos dados. Na quinta parte, encontra-se uma breve seção que apresenta os trabalhos relacionados e por fim, na última parte, encontram-se as considerações finais, limitações da pesquisa e sugestão para trabalhos futuros.

\section{FUNDAMENTAÇÃO TEÓRICA}

Nos tópicos que seguem serão discutidos os principais pressupostos teóricos referentes aos modelos de mensuração da satisfação dos clientes, a aplicação dos modelos de satisfação na literatura nacional e ao ecommerce. 


\subsection{Modelos de mensuração da satisfação dos clientes}

Neste tópico serão relacionados os modelos de mensuração da satisfação de clientes que foram utilizados para embasar este estudo, que são: o método SERVQUAL, o Método SERVPERF, os Barômetros Nacionais (Modelo Sueco, Modelo Americano, Modelo Europeu) e o Modelo Brasileiro Quali Quanti, conforme apresentados a seguir.

A escala SERVQUAL é um método baseado no paradigma da desconformidade, ou seja, parte do pressuposto que o cliente tem expectativas em relação ao desempenho do produto antes de comprá-lo. 0 objetivo do instrumento é medir a satisfação dos clientes com a qualidade do serviço prestado pelas organizações. Para isso, os autores do modelo, Parasuraman, Zeithaml e Berry (1988) consideram apropriado avaliá-la através da subtração do escore de percepções do escore de expectativas.

Nesse instrumento, a qualidade dos serviços é medida sob a ótica de cinco dimensões de qualidade que se desdobram em vinte e dois atributos necessários para realizar a mensuração da satisfação dos usuários com a qualidade dos serviços (SALOMI; MIGUEL; ABACKERLI, 2005).

De acordo com Salomi, Miguel e Abackerli (2005), a definição das cinco dimensões da qualidade dessa escala são: Tangibilidade, que se refere à aparência das instalações, equipamentos, pessoal envolvido e material de comunicação; Confiabilidade, que diz respeito à habilidade de prestar o serviço com exatidão; Presteza, que está relacionada à disposição em ajudar os clientes e fornecer o serviço com presteza e prontidão (prontidão nas providências e respostas); Segurança, que possui relação com o conhecimento dos funcionários e suas habilidades em demonstrar confiança e Empatia, que indica o grau de cuidado e atenção pessoal dispensado aos clientes.

Outra linha de pensamento acerca da mensuração da satisfação é o Modelo baseado na Performance Percebida, o qual supõe que a mensuração da satisfação e da performance percebida pode ser obtida mediante a avaliação da percepção de performance. Faz uso dessa metodologia o SERVPERF, que surgiu a partir do aprimoramento do SERVQUAL, possuindo, portanto, o objetivo de minimizar algumas limitações trazidas pelo modelo original. 0 instrumento desenvolvido por Cronin e Taylor (1992) considera que a qualidade dos serviços é resultante da percepção dos clientes com o desempenho do serviço e não resultado da diferença entre expectativa e desempenho, como ocorre no SERVQUAL.

Cronin e Taylor (1992) excluíram do SERVPERF as cinco dimensões de qualidade presentes no SERVQUAL por considerarem-nas questionáveis, mantendo, no entanto, os 22 atributos, os quais, segundo eles, estão bem fundamentados e representam satisfatoriamente a qualidade em serviços. Além disso, os autores comprovaram através de um estudo empírico a eficácia do método sem que fossem inseridas as cinco dimensões propostas no SERVQUAL. Segundo os autores, através da pesquisa realizada, ficou evidente que a escala de itens que definem a qualidade em serviços em um segmento pode ser diferente em outro.

Assim, a principal tese defendida pelos autores do SERVPERF é que a qualidade pode ser mensurada apenas através da avaliação das percepções dos clientes, visto que esta é um antecedente da satisfação e possui grande influência nas intenções de compras.

Além dessas metodologias apresentadas, existem na literatura outras abordagens de avaliação da satisfação que se baseiam na percepção dos clientes, como o Método Brasileiro Quali Quanti. Esse modelo pressupõe que a pesquisa de satisfação deve ser desenvolvida em duas etapas: na primeira levantam-se os atributos, ou seja, os elementos responsáveis pela satisfação dos clientes, e na segunda etapa é mensurada a satisfação dos clientes sobre esses atributos.

O Método Brasileiro de Pesquisa de Satisfação de Clientes foi desenvolvido pelos brasileiros Rossi e Slongo (1998). Para os autores, as pesquisas de satisfação devem avaliar atributos que o cliente realmente considera importantes, ou seja, que são capazes de provocar a satisfação ou insatisfação. Assim, esse modelo está dividido em duas fases. A primeira fase compreende uma pesquisa de caráter exploratório e qualitativa, que tem como objetivo encontrar indicadores de satisfação que serão a base da coleta de dados da segunda fase da pesquisa. A segunda fase possui natureza descritiva e quantitativa e destina-se propriamente a medir o grau de satisfação dos clientes.

Outra abordagem encontrada com frequência na literatura quando 0 assunto é a satisfação dos clientes são os Modelos apoiados em Métodos de Equações Estruturais, popularmente conhecidos como 
Barômetros Nacionais, como o Swedish Customer Satisfaction Barometer(SCSB), desenvolvido na Suécia por Fornell (1992), o American Customer Satisfaction Index (ACSI), proposto por Fornell et al. (1996) e o Modelo Europeu, elaborado pela Mouvement Français pourla Qualité(MFQ), em 1996. Esses modelos são baseados na percepção dos clientes quanto ao pós-consumo e a prospecção à lealdade. Não detalharemos mais a fundo esses modelos em razão de que se percebeu que eles possuem uma abordagem de análise da satisfação diferente dos demais modelos, não sendo viável sua utilização para a elaboração do instrumento proposto.

Os modelos apresentados neste tópico possuem as mais diversas percepções para avaliação da satisfação dos clientes. No entanto, para que a mensuração da satisfação dos clientes seja contundente e eficaz para auxiliar os gestores na tomada de decisão, é necessário considerar as peculiaridades de cada segmento. Nesse sentido, considerando o propósito desse trabalho, o tópico seguinte aborda al gumas definições básicas a respeito do comércio eletrônico, com o intuito de auxiliar na compreensão de suas especificidades para fomentar a construção desse estudo.

\subsection{Aplicação dos modelos de satisfação na literatura nacional}

$\mathrm{Na}$ literatura nacional, muitos estudos podem ser encontrados sobre a satisfação dos clientes. Nesta seção são apresentados alguns estudos encontrados através de busca no Portal de Periódicos da CAPES, Portal de Revista SciELO (Scientific Electronic Library On-line) e Revista Brasileira de Marketing - REMark. As metodologias com maior número de publicações encontradas são: Modelo SERVQUAL, Modelo SERVPERF, Modelo Sueco (SCSI) Modelo Americano (ACSI), Modelo Europeu (ECSI).

Nos artigos encontrados, alguns autores se restringiram apenas em replicar determinado modelo para avaliar a satisfação, enquanto outros fizeram adaptações nos modelos para avaliar a qualidade dos serviços de um ramo específico. Os autores Salomi, Miguel e Abackerli (2005) realizaram uma pesquisa para avaliar a qualidade do serviço interno de uma empresa do ramo Industrial Mecânico. Para isso, compararam o SERVQUAL, SERVPERF, SERVQUAL ponderado e SERVPERF ponderado.

Freitas e Cozendey (2008) adaptaram o modelo SERVPERF para avaliar a qualidade dos serviços hospitalares. Urdan e Rodrigues (1999) utilizaram o ACSI para analisar a satisfação dos clientes de indústrias automobilísticas de Belo Horizonte/MG. Lopes, Pereira e Vieira (2009) realizaram uma pesquisa com o objetivo de comparar os modelos ACSI e ECSI de satisfação do cliente, identificando qual possui maior aderência para medir a satisfação de clientes de empresas prestadoras de serviços da região Centro-Oeste de MG. Leite e Gonçalves Filho (2007) avaliaram a satisfação utilizando o ECSI em uma empresa brasileira fornecedora de sistemas de informação.

Por último, Marchetti e Prado (2004) propuseram um modelo para avaliar a satisfação no setor elétrico brasileiro utilizando como base o SCSB e o ACSI. Em 2001, os mesmos autores publicaram um artigo no qual discorrem sobre os métodos de avaliação da Satisfação do Consumidor e da Qualidade Percebida e dialogam sobre as vantagens e desvantagens de cada um dos modelos.

\subsection{E-Commerce}

O e-commerce (comércio eletrônico) pode ser definido como o "intercâmbio ou a compra e venda de produtos e serviços por meios eletrônicos" (GORDON; GORDON, 2013, p. 130). Para sua viabilização, utiliza-se de forma intensa a aplicação das tecnologias de comunicação e de informação com o objetivo de que a cadeia de negócios possa ser realizada no ambiente eletrônico (ALBERTIN, 2000).

De acordo com Limeira (2003) as formas com que o comércio eletrônico se desenvolve na internet variam de acordo com o tipo de troca, produtos, serviços e informações que são transacionados neste canal, das quais se destacam quatro modalidades mais comuns: Business-to-Business(B2B), Consumer-to-Consumer (C2C), Consumer-to-Business (C2B), Business-to-Consumer(B2C).

No comércio eletrônico B2C, ou empresa para consumidor, as transações comerciais ocorrem da empresa para o consumidor final. A forma mais comum de B2C envolve sites que centralizam produtos de diversos fornecedores, equivalendo a shopping centers eletrônicos por possuírem uma grande variedade de itens. Essa categoria representa boa parte do varejo eletrônico (BATISTA, 2004). 
A aplicação do comércio eletrônico em varejo representa grande parte da movimentação do segmento de e-commerce atualmente. Parente (2000, p. 22) traz uma definição de varejo e varejista. Segundo ele

(...) o varejo consiste em todas as atividades que englobam o processo de venda de produtos e serviços para atender a uma necessidade pessoal do consumidor final. 0 varejista é qualquer instituição cuja atividade principal consiste no varejo, isto é, na venda de produtos e serviços para o consumidor final.

De acordo com Stair e Reynolds (2006, p. 298) o varejo eletrônico "é a venda direta de empresas para consumidores por lojas eletrônicas, que são geralmente projetadas com base em um catálogo eletrônico e em um modelo de carrinhos de compras". Porto (2000) complementa que nele a comercializaçáo de produtos e serviços ocorre através de um sistema interativo, decorrente da evolução do marketing direto sendo, portanto, uma modalidade de varejo sem loja.

Os e-consumers, também conhecidos como consumidores eletrônicos, são indivíduos que efetuam compras por meio da internet. Dessa forma, com o acentuado crescimento desse tipo de consumidor, tornase necessário compreender seus comportamentos como forma de amparar as empresas com informações que Ihes permitam tomar decisões precisas na hora de realizar vendas on-line (PEREIRA et al., 2011).

No ano de 2016, de acordo com o Relátorio Webshoppers, publicado anualmente pela E-Bit - empresa especializada em informações do comércio eletrônico - apesar do cenário econômico ser considerado desfavorável, o Comércio Eletrônico movimentou $\mathrm{R} \$ 41,3$ bilhões, atingindo uma alta nominal de 15,3\% em relação ao faturamento obtido em 2014 , que foi de $R \$ 35,8$ bilhões. Os pedidos on-line neste ano chegaram a 106,2 milhões, elevando-se em 3\% em relação ao ano anterior e 39,1 milhões de consumidores virtuais realizaram pelo menos uma compra em 2016 (E-BIT, 2016).

0 uso de smartphonese tablets vem influenciando diretamente no comportamento do consumidor virtual. De acordo com o relatório divulgado pela E-Bit, em 2016, o acesso via dispositivos móveis em lojas virtuais no Brasil foi de $30 \%$ e $1 / 3$ do tempo gasto pelos consumidores navegando nas lojas virtuais é realizado com smartphonesou tablets (E-BIT, 2016). Assim, esses dados expressam a importância de que as lojas virtuais estejam cada vez mais acessíveis, através de aplicativos específicos para aparelhos portáteis, para que possam ser visitadas por qualquer pessoa e de qualquer lugar.

\section{METODOLOGIA}

Para atender o objetivo desse estudo, a metodologia da pesquisa realizada foi dividida em duas etapas, sendo a primeira classificada como qualitativa e exploratória e a segunda, quantitativa e descritiva.

A etapa qualitativa e exploratória teve o objetivo de levantar, identificar e analisar as características dos modelos de mensuração da satisfação escolhidos para amparar este estudo, mediante realização de pesquisa bibliográfica. A partir disso, foi possível utilizar algumas características destes modelos para propor o instrumento para mensurar a satisfação dos clientes de e-commerce.

Para auxiliar na estruturação do instrumento adotou-se a estratégia defendida por Rossi e Slongo (1998), os quais propuseram no método Brasileiro Quali Quanti, uma etapa qualitativa e exploratória para levantar os atributos considerados importantes para a avaliação da satisfação dos clientes. Para esta etapa da pesquisa, a definição da amostra foi caracterizada como não probabilística por julgamento que, segundo Malhotra (2012), caracteriza-se como uma forma de amostragem por conveniência em que os elementos da população são escolhidos deliberadamente com base no julgamento do próprio pesquisador.

A coleta das informações se deu por meio de entrevista pessoal e em profundidade. A entrevista em profundidade é um método de obtenção de dados qualitativos, e constitui-se de uma forma não estruturada, direta e pessoal de obter informações (MALHOTRA, 2012).

As entrevistas ocorreram durante o mês de março de 2016. Para auxiliar este processo foi utilizado um roteiro de entrevista semiestruturado, composto por 14 perguntas abertas que possibilitaram que, ao longo da conversa, o entrevistado fosse instigado a relatar experiências e situações pertinentes ao tema como forma de enriquecer a coleta dos dados.

A técnica de análise dos dados adotada foi análise de conteúdo, a qual compreende a "descrição objetiva, sistemática e quantitativa do conteúdo manifesto de uma comunicação" (MALHOTRA, 2012, p.159). 
Nesse sentido, as entrevistas pessoais evidenciaram as percepções dos usuários quanto aos aspectos que influenciam suas compras e contribuem para a sua satisfação na internet.

Os relatos dos entrevistados foram descritos de forma a se identificar quais as ocorrências eram mais comuns entre eles e permitiram visualizar os aspectos que provocam a satisfação ou insatisfação nas compras pela internet. Assim, as entrevistas auxiliaram a composição das dimensões e os atributos do instrumento de mensuração da satisfação dos clientes de e-commerce proposto neste estudo.

Após a formulação do instrumento, na segunda etapa da pesquisa, caracterizada como quantitativa, ocorreu, de forma empírica, a aplicação prática do instrumento de mensuração de satisfação de clientes de $e$ commerce $\mathrm{B} 2 \mathrm{C}$ proposto por este estudo, conforme questionário elaborado para a coleta de dados.

A pesquisa foi aplicada em âmbito nacional adotando-se a metodologia do survey. De acordo com Prodanov e Freitas (2013, p. 57), o levantamento do tipo survey "ocorre quando envolve a interrogação direta das pessoas cujo comportamento desejamos conhecer através de algum tipo de questionário".

Para a pesquisa proposta, os sujeitos foram selecionad os entre clientes de um e-commerce específico de varejo on-line que figura entre os maiores do Brasil em faturamento anual. A amostra escolhida para a realização da pesquisa caracteriza-se como probabilística infinita, calculada através da equação 2, proposta por Martins (2011, p. 45).

$$
n=\frac{z^{2} \times p \times q}{e^{2}}
$$

Onde:

$\mathrm{n}=$ amostra que será calculada

$\mathrm{z}=$ valor crítico

$\mathrm{e}=$ erro amostral

p.q $=$ porcentagem pelo qual o fenômeno ocorre.

Para a aplicação do cálculo da amostra, o nível de confiança que foi considerado é de $95 \%$, o que representa Z igual a 1,96. 0 valor utilizado para o p e para o q foi 0,5. Já o erro amostral foi de $5 \%$. Dessa forma, 384 era o número mínimo de questionários a serem coletados, de acordo com o cálculo.

0 instrumento utilizado para a coleta de dados foi um questionário, viabilizado via Google Docs e disponibilizado aos consumidores durante os dias 7 a 25 de Abril de 2017. A divulgação dos questionários se deu através de canais de internet, como redes sociais e correio eletrônico.

Os itens que avaliavam diretamente a satisfação foram submetidos à análise descritiva dos dados, que de acordo com Silvestre $(2007$, p. 4) "é constituída pelo conjunto de métodos destinado à organização e a descrição dos dados através de indicadores sintéticos ou sumários". Nesse caso, foi empregado o cálculo da média aritmética (escore médio) e o cálculo de porcentagem dos dados. Para avaliar a confiabilidade do instrumento, calculou-se o Alfa de Cronbach para cada uma das dimensões do instrumento. Todos os dados coletados na pesquisa foram tabulados com o auxílio da ferramenta Excel ${ }^{\circ}$

\section{ANÁLISE DOS DADOS}

Este capítulo tem o objetivo de demonstrar os procedimentos adotados nas duas etapas em que a pesquisa foi dividida, a primeira de caráter qualitativo e exploratório e a segunda de caráter quantitativo descritivo, e os resultados alcançados em cada uma delas.

\subsection{Etapa qualitativa: proposta do modelo de mensuração}

Este tópico teve como objetivo analisar os modelos de satisfação dos clientes elencados na fundamentação teórica e, a partir disso, identificar as características mais aderentes de cada um deles para a 
estruturação do instrumento de mensuração da satisfação dos clientes de e-commerce B2C, conforme a proposta deste estudo.

As escalas SERVQUAL e SERVPERF foram concebidas para a mensuração da qualidade de serviços. Dessa forma, diante do fato do e-commerce tratar-se de varejo on-line e não de uma prestação de serviços prop riamente dita, a utilização integral dessas escalas pode ser descartada. No entanto, considera-se relevante para o estudo agregar ao instrumento proposto algumas avaliações sugeridas por esses dois modelos, visto que e-commerce, como todo negócio, possui serviços agregados em suas transações.

O instrumento SERVQUAL é composto por cinco dimensões (Aspectos Tangíveis, Confiabilidade, Presteza, Segurança e Empatia). Para que o instrumento contemplasse mais a fundo as necessidades do comércio eletrônico, foi necessário fazer algumas adequações nas nomenclaturas dessas dimensões. É comum encontrarmos na literatura da área autores que fizeram adaptações de escalas com o objetivo de adequá-las especificamente para a avaliação da qualidade de determinado serviço. Como Freitas e Cozendey (2008) que adaptaram a estrutura SERVPERF para avaliarem serviços hospitalares, e Ferraz Júnior e Salles (2010) que apresentaram um estudo em que fizeram adequações na estrutura do instrumento SERVQUAL para avaliação da percepção de desempenho de serviços de manutenção em uma autarquia federal brasileira.

Dessa forma, tendo em vista que o objetivo deste estudo é desenvolver um instrumento híbrido, optou-se pela utilização das dimensões Aspectos Tangíveis e Confiabilidade, que estão presentes no modelo SERVQUAL. As dimensões Segurança, Presteza e Empatia foram transformadas em uma única dimensão denominada "Segurança e Atendimento", pois esse agrupamento mostrou maior aderência com a proposta do estudo, devido à similaridade dessas três dimensões, as quais referem-se ao nível de atendimento das necessidades dos clientes virtuais. Assim sendo, cabe salientar que a denominação "segurança" dessa dimensão não se refere à segurança oferecida pelo aparato tecnológico do site e sim, pela segurança transmitida pelos seus funcionários através do atendimento prestado por eles, em situações de dúvidas ou problemas dos clientes.

No instrumento SERVQUAL, a avaliação da qualidade dos serviços é obtida através da diferença entre a percepção e a expectativa que o consumidor tem dos serviços consumidos. No entanto, no método proposto, optou-se por suprimir a avaliação das expectativas dos clientes, baseando-se na proposta do SERVPERF, a qual defende que a avaliação da qualidade dos serviços pode ser medida apenas através da avaliação da percepção/experiência do cliente com o serviço.

A exclusão da expectativa na configuração do instrumento proposto está amparada em autores que defendem que a expectativa não é um antecedente da satisfação como o SERVQUAL (CRONIN; TAYLOR, 1992), e sugerem que avaliar a qualidade através da diferença entre expectativa e percepção deve ser evitado. Além disso, ao desconsiderar a expectativa, tem-se um método mais rápido e eficiente, pois se reduz em $50 \%$ o número de itens que precisam ser mensurados (CRONIN; TAYLOR, 1992).

O Modelo Brasileiro Quali Quanti também serviu de base para a estruturação do instrumento proposto para o e-commerce. Através da utilização da abordagem qualitativa e exploratória proposta pelo modelo, buscou-se identificar outros aspectos, características e benefícios considerados importantes pelos consumidores para a avaliação de sua satisfação em compras virtuais.

Aplicando essa abordagem, procederam-se entrevistas em profundidade com seis usuários habituais de lojas de e-commerce para levantar os atributos e itens de satisfação que eles consideravam importantes. De acordo com as entrevistas, surgiram novos atributos que foram agrupados em três novas dimensões: Disponibilidade; Preços e Condições de Pagamento; e Serviços Pós-Venda, conforme apresentado a seguir.

A dimensão "Disponibilidade" refere-se à prontidão pela qual os itens são oferecidos para o cliente. A dimensão "preço e condições de pagamento" refere-se à política de preços dos produtos e ao valor do frete, bem como formas de pagamento e descontos oferecidos. Os aspectos relacionados aos serviços com que o cliente tem acesso após a realização da compra também foram citados pelos entrevistados, originando a dimensão "Serviços Pós-Venda".

No quadro 1 apresenta-se o instrumento de satisfação proposto neste estudo. 
Quadro 1 - Instrumento proposto para a mensuração da satisfação de cliente de e-commerce.

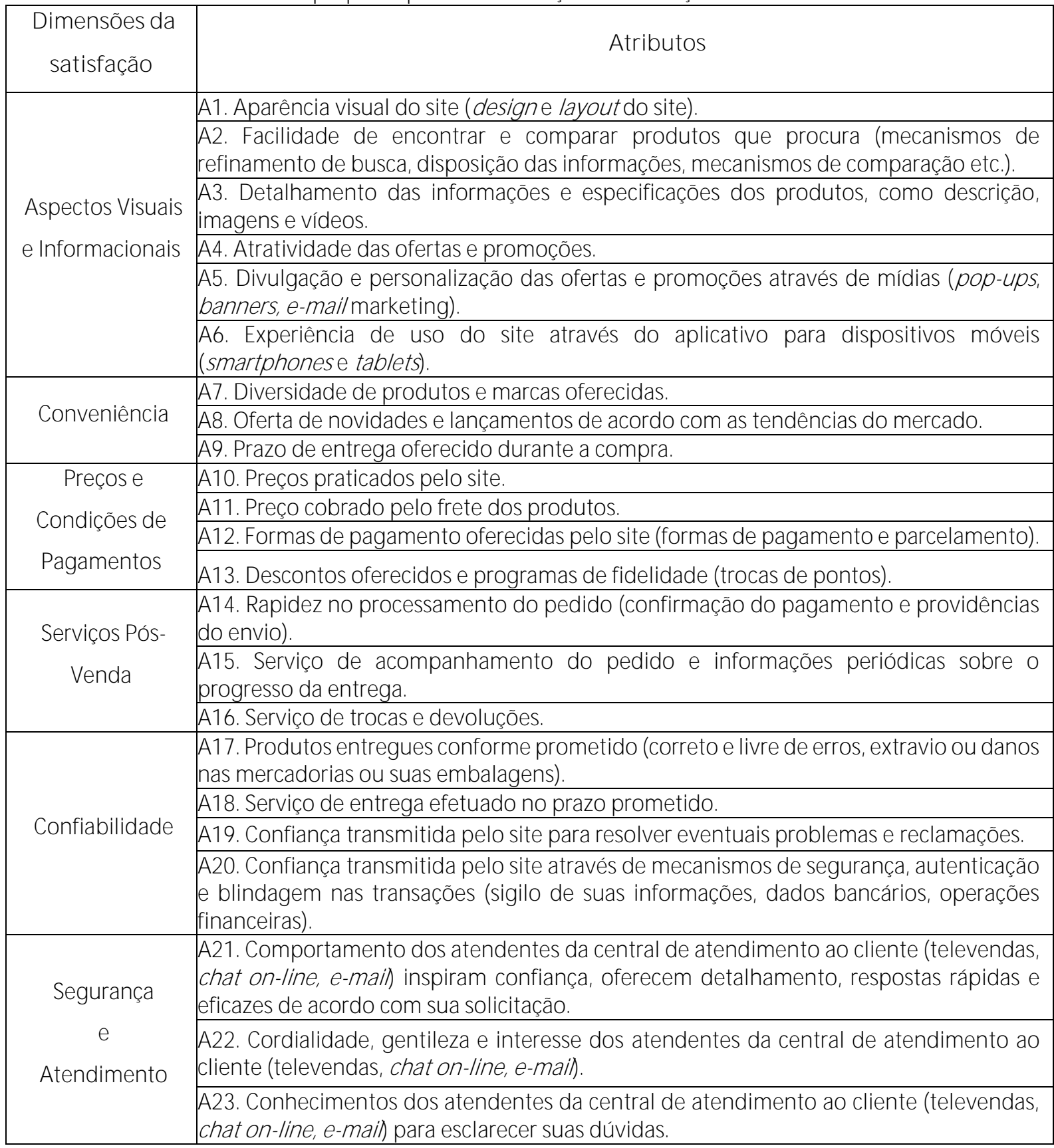

Fonte: Elaborado pelos autores (2016).

0 instrumento preliminar é composto por 23 atributos (características) agrupados em seis dimensões da satisfação: Aspectos Visuais e Informacionais (A1 a A6), Conveniência (A7 a A9), Preços e Condições de Pagamentos (A10 a A13), Serviços Pós-Venda (A14 a A16), Confiabilidade (A17 a A20), Segurança e Atendimento (A21 a A23).

As entrevistas em profundidade realizadas nessa fase permitiram identificar quatro elementos principais que influenciam a compra na internet e contribuem para a satisfação dos clientes, das quais se destacam: usabilidade e navegabilidade do site; preço; confiabilidade e atendimento.

A aparência do site e os elementos relacionados à sua usabilidade e navegabilidade contribuem para que o usuário tenha uma experiência agradável e não fique desestimulado a comprar. 0 preço é a grande variável desencadeadora da venda; o cliente procura sempre maximizar o valor de suas transações e a sensação de ter realizado um bom negócio. 0 terceiro elemento observado nas entrevistas refere-se à confiabilidade: sem este elemento nenhuma transação ocorre no ambiente virtual. Por fim, o quarto elemento refere-se ao 
atendimento prestado pelo site através dos canais de interatividade (televendas, e-mail e chat on-line). A disponibilização desses canais confere ao cliente maior confiança com a loja e a sensação de ter a quem recorrer em situações de dúvidas ou problemas.

\subsection{Etapa quantitativa}

Nesta etapa apresentam-se os resultados obtidos através da validação empírica do instrumento proposto em um dos maiores e-commerce de varejo on-line do Brasil. Participaram da pesquisa clientes que realizaram compras nos últimos doze meses. Após a divulgação do questionário, através de e-mail e redes sociais, obteve-se 384 respostas. Em relação ao perfil da amostra, pode-se destacar que $56,25 \%$ são mulheres, $82,6 \%$ têm até 30 anos, $75,5 \%$ são solteiros, 59,9\% estão cursando ensino superior, $48,7 \%$ são estudantes e $77,1 \%$ possuem renda familiar de até $\mathrm{R} \$ 6.400,00$.

\subsubsection{Teste da Confiabilidade do instrumento}

Para testar o grau de confiabilidade de cada dimensão do questionário, utilizou-se o coeficiente Alfa de Cronbach. Segundo Cortina (1993) este índice mede a consistência interna de uma escala, indicando o grau de correlação entre os itens do instrumento. 0 valor do Alfa de Cronbach varia de 0 a 1 . Para ser considerado aceitável, seu valor mínimo deve ser 0,70 STREINER (2003).

O Alfa de Cronbach foi calculado de acordo com a equação 2, proposta por Leontitsis e Pagge (2007).

$$
\alpha=\frac{\mathbf{k}}{\mathbf{k}-1}\left[\frac{\sigma_{\mathbf{\tau}}^{2}-\Sigma_{\mathbf{i}=1}^{\mathbf{k}} \sigma_{\mathbf{i}}^{2}}{\sigma_{\tau}^{2}}\right]
$$

A tabela 1 apresenta o Alfa de Cronbach para cada uma das dimensões do instrumento avaliadas a partir das percepções dos clientes.

Tabela 1 - Teste da confiabilidade do questionário

\begin{tabular}{|c|c|c|c|}
\hline Dimensão & Atributos & $\begin{array}{c}\text { Alfa de Cronbach } \\
\text { (se o atributo for excluído) }\end{array}$ & $\begin{array}{l}\text { Alfa de Cronbach } \\
\text { da dimensão }\end{array}$ \\
\hline \multirow{6}{*}{$\begin{array}{c}\text { Aspectos Visuais } \\
\text { e } \\
\text { Informacionais }\end{array}$} & A1 & 0,7125 & \multirow{6}{*}{0,7380} \\
\hline & $\mathrm{A} 2$ & 0,7068 & \\
\hline & A3 & 0,6897 & \\
\hline & A4 & 0,6774 & \\
\hline & A5 & 0,6876 & \\
\hline & A6 & 0,7273 & \\
\hline \multirow{3}{*}{ Conveniência } & A7 & 0,5106 & \multirow{3}{*}{0,6470} \\
\hline & A8 & 0,4658 & \\
\hline & A9 & 0,6872 & \\
\hline \multirow{5}{*}{$\begin{array}{l}\text { Preços e Condições de } \\
\text { Pagamentos }\end{array}$} & A10 & 0,5531 & \multirow{5}{*}{0,6684} \\
\hline & Al1 & 0,6210 & \\
\hline & A12 & 0,6165 & \\
\hline & A13 & 0,6165 & \\
\hline & A14 & 0,5844 & \\
\hline \multirow[t]{4}{*}{ Serviços Pós-Venda } & A15 & 0,5513 & \multirow[t]{3}{*}{0,7160} \\
\hline & A16 & 0,7132 & \\
\hline & A17 & 0,7430 & \\
\hline & A18 & 0,7507 & \multirow{4}{*}{0,8095} \\
\hline \multirow{2}{*}{ Confiabilidade } & A19 & 0,7775 & \\
\hline & $\mathrm{A} 20$ & 0,7712 & \\
\hline \multirow{3}{*}{$\begin{array}{c}\text { Segurança } \\
\text { E Atendimento }\end{array}$} & A21 & 0,8652 & \\
\hline & A22 & 0,8676 & \multirow[t]{2}{*}{0,9121} \\
\hline & A23 & 0,8872 & \\
\hline
\end{tabular}


Analisando-se os coeficientes obtidos, percebe-se que quatro dimensões apresentam alfas considerados satisfatórios, "Aspectos Visuais e Informacionais", "Serviço pós-venda", "Confiabilidade" e "Segurança e Atendimento" com Alfas de Cronbach de 0,74, 0,72, 0,81 e 0,91, nesta ordem. As outras duas dimensões, "Conveniência" e "Preços e Condições de Pagamento" apresentaram coeficientes abaixo de 0,7, sugerindo a falta de consistência interna dos atributos medidos nessas dimensões. A falta de consistência interna ocorre quando os atributos medidos em uma mesma dimensão não estão correlacionados, ocasionando oscilação entre as respostas dos atributos.

\subsubsection{Análise descritiva dos dados}

Para a avaliação da satisfação dos clientes segundo os atributos do instrumento proposto, utilizou-se uma Escala Likert de cinco pontos, sendo composta por totalmente insatisfeito (1), insatisfeito (2), satisfeito (4) e totalmente satisfeito (5), possuindo uma escala intermediária entre elas, nem satisfeito, nem insatisfeito (3).

A tabela 2 mostra uma síntese das avaliações da satisfação obtidas na pesquisa, apresentando 0 escore médio de cada um dos atributos e o escore médio de cada uma das dimensões.

Tabela 2 - Resultados gerais da satisfação (dimensão e atributo)

\begin{tabular}{|c|c|c|}
\hline Dimensão & Atributo & Escore médio \\
\hline $\begin{array}{l}\text { Aspectos Visuais e } \\
\text { Informacionais }\end{array}$ & $\begin{array}{l}\text { A1 } \\
\text { A2 } \\
\text { A3 } \\
\text { A4 } \\
\text { A5 } \\
\text { A6 }\end{array}$ & $\begin{array}{l}3,88 \\
3,84 \\
3,78 \\
3,63 \\
3,46 \\
3,20\end{array}$ \\
\hline \multicolumn{2}{|c|}{ Satisfação da dimensão } & 3,63 \\
\hline Conveniência & $\begin{array}{l}\text { A7 } \\
\text { A8 } \\
\text { A9 }\end{array}$ & $\begin{array}{l}3,92 \\
3,77 \\
3,91\end{array}$ \\
\hline \multicolumn{2}{|c|}{ Satisfação da dimensão } & 3,87 \\
\hline $\begin{array}{c}\text { Preços e Condições de } \\
\text { Pagamentos }\end{array}$ & $\begin{array}{l}\text { A10 } \\
\text { A11 } \\
\text { A12 } \\
\text { A13 }\end{array}$ & $\begin{array}{c}3,77 \\
3,2 \\
4,07 \\
3,18\end{array}$ \\
\hline \multicolumn{2}{|c|}{ Satisfação da dimensão } & 3,56 \\
\hline Pós-venda & $\begin{array}{l}\text { A14 } \\
\text { A15 } \\
\text { A16 }\end{array}$ & $\begin{array}{l}3,77 \\
3,74 \\
3,23 \\
\end{array}$ \\
\hline \multicolumn{2}{|c|}{ Satisfação da dimensão } & 3,58 \\
\hline Confiabilidade & $\begin{array}{l}\text { A17 } \\
\text { A18 } \\
\text { A19 } \\
\text { A20 }\end{array}$ & $\begin{array}{l}4,11 \\
4,09 \\
3,49 \\
4,05\end{array}$ \\
\hline \multicolumn{2}{|c|}{ Satisfação da dimensão } & 3,94 \\
\hline Segurança e Atendimento & $\begin{array}{l}\text { A21 } \\
\text { A22 } \\
\text { A23 }\end{array}$ & $\begin{array}{l}3,43 \\
3,43 \\
3,34\end{array}$ \\
\hline \multicolumn{2}{|c|}{ Satisfação da dimensão } & 3,40 \\
\hline
\end{tabular}

De acordo com a tabela, os escores médios dos atributos variaram de 3,18 a 4,11; a maioria mantevese na casa dos três pontos demonstrando que a variação das avaliações oscilou entre indiferentes a muito satisfeitos. Não há escorres médios que demonstrem clientes insatisfeitos ou muito insatisfeitos. Para a maioria 
dos atributos, os clientes consideraram-se indiferentes a satisfeitos, revelando a ausência de serviços de valor superior. Em todos os atributos há margem para melhorias.

As dimensões "Confiabilidade" e "Conveniência" foram as que concentraram melhores avaliações da satisfação, com escore médio $(3,94)$ e $(3,87)$, respectivamente, seguidas de "Aspectos Visuais e Informacionais" $(3,63)$, "Pós-venda" $(3,58)$ e "Preços e Condições de Pagamento" $(3,56)$. A dimensão com a pior avaliação foi "Segurança e Atendimento" (3,48), os atributos avaliados nessa dimensão estão dentre os que obtiveram escore médio da satisfação mais baixo.

Merecem destaque os atributos A12, A17, A18 e A20, os quais obtiveram maior escore médio de satisfação, acima de quatro pontos, denotando cliente satisfeito a muito satisfeito, com escore médio de 4,07, $4,11,4,09,4,05$, nesta ordem.

Os atributos A5 (34,4\%), A6 (55,5\%), A13 (49,2\%), A16 (64,1\%), A19 (43\%), A21 (44,5\%), A22 (48,2\%) e A23 (50,5\%) concentraram percentuais acima de $30 \%$ de respostas de "nem satisfeito, nem insatisfeito" (3), sugerindo que grande parte dos clientes não tinha nenhuma experiência de consumo com esses atributos no momento da pesquisa e, por isso não possuíam parâmetros para expressar algum grau de satisfação ou insatisfação. Isso fez com que os escores médios fossem os mais baixos, próximo a três pontos $(3,46,3,203,18$, $3,23,3,49,3,43,3,43$ e 3,34). Por essas razões, a análise desses atributos foi substancialmente prejudicada, visto que não foi possível obter a real satisfação ou insatisfação dos clientes com esses serviços.

Os atributos que concentraram percentuais de insatisfeitos acima de $10 \%$ foram o A5 com escore médio de 3,46 e 14,3\% de insatisfeitos; A6 com escore médio de 3,20 e 13,5\% de insatisfeitos; A11 com escore médio de 3,20 e 27,1\% de insatisfeitos; A13 com escore médio de 3,18 e 17,4\% de insatisfeitos; e A15 com escore médio de 3,74 e $12,5 \%$ de insatisfeitos. Os demais atributos obtiveram percentuais de insatisfeitos abaixo de $10 \%$, oscilando entre $2,6 \%$ e $9,6 \%$.

Através da aplicação empírica do método, algumas considerações podem ser feitas: inicialmente, salienta-se a necessidade de mudança na escala do instrumento, visto que alguns atributos, por sua natureza, não são necessariamente consumidos por quem efetua compras pela internet, como por exemplo, entrar em contato com o SAC e a utilização do aplicativo do site. Por essa razão, não havia como os clientes expressarem seu estado de satisfação ou insatisfação sem que tivessem alguma experiência de consumo. Assim, sugere-se que na escala haja uma opção como "não sei ou não se aplica".

Além disso, sugere-se uma readequação das dimensões e em alguns atributos avaliados no instrumento para que apresentem uma melhor adequação para avaliação da satisfação dos clientes de $e$ commerce, conforme observado no quadro 2 .

Quadro 2 - Readequação do instrumento proposto

\begin{tabular}{|c|c|}
\hline Dimensão & Atributos \\
\hline \multirow{6}{*}{$\begin{array}{l}\text { Aspectos } \\
\text { visuais } \\
\text { e } \\
\text { informacionais }\end{array}$} & Aparência visual do site (design e layout do site). \\
\hline & $\begin{array}{l}\text { Facilidade de encontrar e comparar produtos que procura (mecanismos de } \\
\text { refinamento de busca, disposição das informações, mecanismos de comparação } \\
\text { etc.). }\end{array}$ \\
\hline & $\begin{array}{l}\text { Detalhamento das informações e especificações dos produtos, como descrição, } \\
\text { imagens e vídeos. }\end{array}$ \\
\hline & Atratividade das ofertas e promoções. \\
\hline & $\begin{array}{l}\text { Divulgação e personalização das ofertas e promoções através de mídias (pop-ups, } \\
\text { banners, e-mail marketing). }\end{array}$ \\
\hline & $\begin{array}{l}\text { Experiência de uso do site através do aplicativo para dispositivos móveis } \\
\text { (smartphonese tablets). }\end{array}$ \\
\hline \multirow{3}{*}{ Conveniência } & Diversidade de produtos e marcas oferecidas. \\
\hline & Oferta de novidades e lançamentos de acordo com as tendências do mercado. \\
\hline & Disponibilidade de estoque dos produtos. \\
\hline \multirow{3}{*}{$\begin{array}{l}\text { Preços } \\
\text { e } \\
\text { Pagamentos }\end{array}$} & Preços praticados pelo site. \\
\hline & Formas de pagamento oferecidas pelo site (formas de pagamento e parcelamento). \\
\hline & Preço cobrado pelo frete dos produtos. \\
\hline
\end{tabular}




\begin{tabular}{|c|c|}
\hline & Descontos oferecidos e programas de fidelidade (trocas de pontos). \\
\hline \multirow{4}{*}{$\begin{array}{l}\text { Serviços de } \\
\text { entrega }\end{array}$} & $\begin{array}{l}\text { Rapidez no processamento do pedido (confirmação do pagamento e providências } \\
\text { do envio). }\end{array}$ \\
\hline & $\begin{array}{l}\text { Serviço de acompanhamento do pedido, informações periódicas sobre o progresso } \\
\text { da entrega. }\end{array}$ \\
\hline & Prazo de entrega oferecido durante a compra. \\
\hline & $\begin{array}{l}\text { Qualidade do atendimento da empresa responsável pela entrega (transportadora, } \\
\text { Correios). }\end{array}$ \\
\hline \multirow{4}{*}{ Confiabilidade } & $\begin{array}{l}\text { Produtos entregues conforme prometido (correto e livre de erros, extravio ou danos } \\
\text { nas mercadorias ou suas embalagens). }\end{array}$ \\
\hline & Serviço de entrega efetuado no prazo prometido. \\
\hline & Confiança transmitida pelo site para resolver eventuais problemas e reclamações. \\
\hline & $\begin{array}{l}\text { Confiança transmitida pelo site através de mecanismos de segurança, autenticação } \\
\text { e blindagem nas transações (sigilo de suas informações, dados bancários, operações } \\
\text { financeiras). }\end{array}$ \\
\hline \multirow{4}{*}{$\begin{array}{l}\text { Segurança } \\
\text { e } \\
\text { Atendimento }\end{array}$} & $\begin{array}{l}\text { Comportamento dos atendentes do serviço de atendimento ao cliente (televendas, } \\
\text { chat on-line, e-mail) inspira confiança, oferecem detalhamento, respostas rápidas e } \\
\text { eficazes de acordo com sua solicitação. }\end{array}$ \\
\hline & $\begin{array}{l}\text { Cordialidade, gentileza e interesse dos atendentes do serviço de atendimento ao } \\
\text { cliente (televendas, chat on-line, e-maih). }\end{array}$ \\
\hline & $\begin{array}{l}\text { Conhecimentos dos atendentes do serviço de atendimento ao cliente (televendas, } \\
\text { chat on-line, e-mail) para esclarecer suas dúvidas. }\end{array}$ \\
\hline & Agilidade e bom atendimento recebido para realizar Trocas e devolução. \\
\hline
\end{tabular}
Fonte: Elaborado pelos autores (2017).

Conforme 0 quadro, as dimensões "Aspectos Visuais e Informacionais", "Preços e Condições de Pagamentos" e "Confiabilidade" foram mantidas sem alterações em seus atributos.

Na dimensão "Conveniência" alguns de seus atributos foram alterados, "prazo de entrega oferecido durante a compra" foi retirado dessa dimensão por não apresentar similaridade com os demais. E foi agregado um novo atributo, "Disponibilidade de estoque dos produtos", devido à falta de estoque no momento da realização da compra ser um fator frustrante para os clientes.

A dimensão "Serviço Pós-Venda" foi extinta, e no lugar foi criada a dimensão "Serviços de entrega", por este serviço ser considerado uns principais fatores relacionados à satisfação em compras na internet. Compõe esta dimensão dois atributos que pertenciam anteriormente à dimensão "Serviço Pós-venda" ("Rapidez no processamento do pedido" e "Serviço de acompanhamento do pedido"), e agregou-se o atributo "Prazo de entrega oferecido durante a compra", que antes estava presente na dimensão "Conveniência" e também o atributo "Qualidade do atendimento da empresa responsável pela entrega", visto que o serviço prestado durante a entrega é capaz de refletir boas ou más impressões da loja virtual.

A dimensão "Segurança e Atendimento" foi mantida, e agregou-se o atributo "Agilidade e bom atendimento recebido para realizar Trocas e devolução", que estava presente anteriormente na dimensão "Serviço Pós-venda". Considerando que esse tipo de serviço demanda de atendimento do SAC para ser solicitado, considerou-se mais adequado fazer essa alteração.

Com os resultad os obtidos com a aplicação empírica do instrumento e as adequações propostas neste tópico, sugere-se que o instrumento abrange com efetividade as necessidades do comércio eletrônico no que tange à mensuração da satisfação dos clientes. Além disso, é possível perceber o dinamismo desse segmento e a importância do constante aprimoramento dos serviços oferecidos por ele com o objetivo de oferecer qualidade aos clientes e grandes doses de encantamento.

De modo complementar, verifica-se que o campo de estudo acerca da satisfação de clientes de $e$ commerce é um tema atual, para o qual se observa uma série publicações, mas que ainda demanda por estudos que preencham as lacunas de conhecimento existentes.

Dentre as publicações, podem-se citar os trabalhos de Parasuraman, Zeithaml e Malhotra (2005) e Loiacono (2000). Os autores Parasuraman, Zeithaml e Malhotra (2005) desenvolveram a estrutura e-S-Qual para 
mensurar a qualidade em Varejo Eletrônico. 0 instrumento é composto por quatro dimensões da qualidade (eficiência, disponibilidade do sistema, preenchimento e privacidade) e 22 itens para avaliá-la sob a percepção do consumidor.

Loiacono (2000) propôs um instrumento chamado WEBQUAL para avaliar a qualidade de ambientes virtuais. 0 modelo foi proposto com base em entrevistas com consumidores, estudantes de graduação e designers de sites. O WEBQUAL é composto por quatro construtos (Facilidade de uso, Usabilidade, Interatividade e Relação complementar), 12 dimensões (Adequação de informação para a tarefa; Interatividade; Confiabilidade; Tempo de resposta; Facilidade de compreensão; Intuitividade; Atratividade visual; Inovação; Apelo emocional; Imagem consistente; Operação on-line; e Comparação com outros canais) e 36 questões para avaliar a qualidade do serviço virtual sob a ótica do consumidor.

Pode-se considerar que tanto o e-S-Qual como o WEBQUAL são estudos pioneiros na área mensuração da qualidade de serviços virtuais, mais especificamente de e-commerce. Percebe-se que existem algumas similaridades entre estas duas ferramentas e o instrumento proposto neste trabalho, como por exemplo, a relevância dada à confiabilidade nas transações on-line. Essa dimensão, sem dúvida, é uma das mais relevantes para a decisão da compra, pois muitos consumidores virtuais ainda possuem insegurança para efetivar transações em ambientes virtuais, principalmente por medo de não terem sua privacidade e segurança de informações resguardadas. Aspectos como facilidade de uso do site, interatividade, usabilidade e disponibilidade dos serviços também são aspectos comuns nos três modelos. Entre as diferenças observadas entre os modelos e-S-Qual e WEBQUAL e o modelo proposto neste trabalho, pode-se citar o que o último considera as dimensões "preço e pagamento" e "serviços de entrega", enquanto os demais não utilizam estes indicadores para a mensuração da qualidade.

\section{CONSIDERAÇÕES FINAIS}

Esta pesquisa propôs um instrumento de mensuração do nível de satisfação de clientes do segmento de e-commerce do tipo Bussiness-to-Consumer (B2C) baseado nas características dos modelos de satisfação existentes. Para isso, inicialmente pesquisou-se algumas das principais metodologias existentes para a mensuração da satisfação de clientes, a saber: escala SERVQUAL, escala SERVPERF, os Barômetros Nacionais (Sueco, Americano e Europeu) e o Modelo Brasileiro Quali Quanti.

Mediante a observação das características de cada um dos modelos, foi adotada a abordagem da performance percebida, conforme propõe o SERVPERF. Além disso, optou-se por adotar a estrutura do SERVQUAL para avaliar alguns aspectos relacionados aos serviços oferecidos no comércio eletrônico. Assim, no instrumento proposto, a formulação de três dimensões foram baseadas no referido modelo. Para se tornar mais aderente à proposta do estudo, as dimensões do SERVQUAL sofreram algumas adaptações em termo de nomenclatura e definição de atributos devido às peculiaridades do segmento.

0 instrumento adotado foi composto por 23 sentenças agrupadas em seis dimensões da satisfação, que são: Aspectos Visuais e Informacionais (6), Conveniência (3), Preço e Condições de Pagamento (4), PósVenda (3), Confiabilidade (4) e Segurança e Atendimento (3). Posteriormente, o modelo foi empiricamente avaliado através de uma pesquisa Survey. Para a aplicação da pesquisa escolheu-se um dos maiores varejos on-line do Brasil. Foram coletados 384 questionários via ferramenta de formulário on-line.

Na sequência, para estimar a confiabilidade do instrumento, calculou-se o Alfa de Cronbach para cada uma das dimensões do modelo. As dimensões "Conveniência" e "Preços e Condições de Pagamento" foram as únicas que apresentaram coeficientes abaixo de 0,7, indicando baixa consistência interna dos atributos medidos nessas dimensões.

A aplicação empírica permitiu constatar que al gumas readequações devem ser feitas no Instrumento com objetivo de melhorar a confiabilidade e o ajustamento com o segmento analisado. Assim, al guns atributos foram reorganizados dentro das dimensões. A dimensão "Serviço Pós-Venda" foi retirada do modelo e foi inserida a dimensão "Serviço de Entrega". As seis dimensões do modelo final são as seguintes: "Aspectos Visuais e Informacionais" (1), "Conveniência" (2), "Preços e Condiçôes de Pagamento" (3), "Serviço de Entrega" (4), "Confiabilidade" (5) e "Segurança e Atendimento" (6). 
Em relação às limitações da pesquisa, pode-se destacar que a divulgação da pesquisa através de $e$ maile redes sociais, direcionou a amostra para um público jovem, não permitindo percepções de uma amostra mais heterogênea de clientes.

Entende-se que a aplicação adequada desse tipo de pesquisa deva ocorrer através do envio do questionário, por e-mail, aos clientes, após a confirmação do recebimento do pedido, assim, suas experiências estariam mais recentes na memória, permitindo que os dados coletados fossem mais contundentes, confiáveis, efetivos e generalizáveis.

Em razão de 0 instrumento proposto ter sofrido alguns ajustes, sugere-se que a pesquisa seja reaplicada a uma amostra maior e mais heterogênea, permitindo percepções de diversos estilos de consumidores. Além disso, a abordagem de validação empírica pode ser alterada, ao invés de aplicar a pesquisa a um e-commerce específico, ela poderia ser empregada a qualquer usuário de lojas virtuais, a fim de confirmar ou refutar os atributos levantados na pesquisa exploratória.

Salienta-se que o instrumento aqui proposto não encerra os estudos acerca do tema. Sua estrutura pode ser depurada empregando-se ferramentas específicas para torná-lo mais confiável. Assim, sugere-se que em trabalhos futuros realize-se análise fatorial para identificar se os atributos agrupados nas dimensões estão de fato correlacionados ou se há necessidade de readequações, tanto das dimensões como dos atributos.

Além disso, para trabalhos futuros recomenda-se a realização de análise de regressão múltipla, para que seja possível determinar a representatividade de cada um dos atributos em relação à satisfação geral da dimensão, em outras palavras, permitiria verificar a importância de cada um dos atributos para determinar a satisfação geral do cliente. Ainda, outras pesquisas qualitativas podem ser realizadas com a finalidade de confirmar, contestar ou readequar a escala proposta, complementando este estudo.

Destaca-se também que a pesquisa sugeriu que alguns dos atributos levantados no instrumento não faziam parte da experiência de uso do cliente, como, por exemplo, o aplicativo para dispositivos móveis e a central de relacionamento com o cliente. Isso serve de subsídio para investigar o porquê isso ocorre, auxiliando na compreensão do comportamento do e-consumer.

Por fim, satisfação dos clientes é um assunto que merece atenção dos gestores, pois está estritamente relacionada aos resultados das empresas e possui relação com a recompra, com a promoção 'boca a boca' positivo da marca e com a lealdade. A importância da satisfação dos clientes faz ainda mais sentido ao levarmos em consideração a frase de Kotler e Keller $(2012$, p.36) "conquistar um novo cliente custa de 5 a 7 vezes mais que manter um atual".

\section{REFERÊNCIAS}

AAKER, David A. Administração estratégica de mercado. Porto Alegre: Bookman, 2012.

ALBERTIN, Alberto Luiz. Comércio eletrônico: Modelos, aspectos e contribuições de sua aplicação. 2. ed. São Paulo: Atlas, 2000.

BAPTISTA, Paulo de Paula; SILVA, Wesley Vieira da; GOSS, Lirian Patty. Qualidade Percebida e seus Impactos sobre a Satisfação, Confiança e Lealdade: um estudo com varejistas eletrônicos de serviços de download gratuito. Revista de Ciências da Administração, Florianópolis, v. 13, n. 30, p. 249-277, maio/ago. 2011. Disponível em: https://periodicos.ufsc.br/index.php/adm/article/view/2175-8069.2008v10n22p80/12653. Acesso em: 20 set. 2017.

BATISTA, Emerson Oliveira. Sistemas de informação. 2. ed. São Paulo: Saraiva, 2004.

CORTINA, J. M. What is coefficient alpha? An examination of theory and applications. Journal of Applied Psychology. v. 78, p. 98-104. 1993.

CRONIN, J. J oseph; TAYLOR, Steven A. Measuring service quality: a reexamination and extension. Journal of Marketing, v. 56, n. 3, p.55-68, J uly 1992. 
E-BIT. Relatório WebShoppers. 2016. Disponível em: http://www.ebit.com.br/webshoppers. Acesso em: 17 maio 2016.

FERRAZ JÚNIOR , J oão Evany ; SALLES, Mara Telles. Adequação do Instrumento SERVQUAL para a Avaliação da Percepção de Desempenho de Serviços de Manutenção - O Caso Inmetro. [2010]. Disponível em: http://www.aedb.br/seget/arquivos/artigos10/412_ARTIGO3Autores.pdf. Acesso em: 17 maio 2016.

FORNELL, Claes. A national customer satisfaction barometer: the swedish experience. Journal Of Marketing, Chicago, v. 56, n. 6, p. 6-21, January. 1992.

FORNELL, Claes et al. The American Customer Satisfaction Index: Nature, Purpose, and Findings. Journalof Marketing, Chicago, v. 60, n. 4, p. 7-18, Oct. 1996.

FREITAS, André Luís Policani; COZENDEY, Manaara lack. Um modelo SERVPERF para avaliação de serviços hospitalares. In: ENCONTRO NACIONAL DE ENGENHARIA DE PRODUÇÃO, 28., 2008, Rio de Janeiro. Anais [...]. Rio de Janeiro: Abepro, 2008. p. 1 - 13.

GORDON, Steven R.; GORDON, J udith R. Sistemas de informação: uma abordagem gerencial. 3. ed. Rio de Janeiro: LTC, 2013.

KOTLER, Philip; KELLER, Kevin L. Administração de marketing. 14. ed. São Paulo: Pearson Education do Brasil Ltda, 2012.

LEITE, Ramon Silva; GONÇALVES FILHO, Cid. Um estudo empírico da aplicação do Índice Europeu de Satisfação de Clientes (ECSI) no Brasil. RAM: Revista de Administração Mackenzie, São Paulo, v. 8, n. 4, p. 178-200, ago. 2007. Disponível em: http://www.redalyc.org/articulo.0a?id=195415184009. Acesso em: 12 out. 2014.

LEONTITSIS, A.; PAGGE, J . A simulation approach on Cronbach's alpha statistical significance. Mathematics and Computers in Simulation. v. 73, p. 336-340. 2007.

LIMEIRA, Tania Maria Vidigal. E-marketing: o marketing na internet com casos brasileiros. São Paulo: Saraiva, 2003.

LOIACONO ,E. T. Webqual: a web site quality instrument. PhD Thesis,University of Georgia, 2000.

LOPES, Humberto Elias Garcia; PEREIRA, Camila Cristina de Paula; VIEIRA, Ana Flávia Santos. Comparação entre os modelos Norte Americano (ACSI) e Europeu (ECSI) de satisfação do cliente: um estudo no setor de serviços. RAM: Revista de Administração Mackenzie, São Paulo, v. 10, n. 1, p. 161-186, jan./fev. 2009. Disponível em: http://www.scielo.br/pdf/ram/v10n1/v10n1a08. Acesso em: 28 ago. 2016.

MALHOTRA, Naresh. Pesquisa de marketing: uma orientação aplicada. 6. ed. Porto Alegre: Bookman, 2012.

MARCHETTI, Renato; PRADO, Paulo H. M. Avaliação da satisfação do consumidor utilizando o método de equações estruturais: um modelo aplicado ao setor elétrico brasileiro. RAC: Revista de Administração Contemporânea, Curitiba. v. 8, n. 4, p.9-32, out./dez. 2004. Disponível em:

http://www.scielo.br/pdf/rac/v8n4/v8n4a02. Acesso em: 03 fev. 2016.

MARTINS, Gilberto de Andrade. Manual para elaboração de monografias e dissertações. 3. ed. São Paulo: Atlas, 2011.

PARASURAMAN, A.; ZEITHAML, Valarie A.; BERRY, Leonard L. SERVQUAL: A Multiple-Item Scale for Measuring Consumer Perceptions of Service Quality. J ournal Of Retailing, New York v. 64, n. 1, p.12-40, 1988.

PARENTE, J uracy. Varejo no Brasil: gestão e estratégia. São Paulo: Atlas, 2000. 
PEREIRA, F. R.; DREKENER, T. L.; OLIVEIRA, M. O. R.; BOBSIN, D. Análise dos fatores que influenciam as compras pela internet. SEMINÁRIOS EM ADMINISTRAÇÃO (SEMEAD), 14., 2011, São Paulo. Anais [...]. São Paulo: FEAUSP, 2011.

PORTO, Roseli Morena. Internet e Varejo Virtual. In:PARENTE, J uracy. Varejo no Brasil: Gestão e Estratégia. São Paulo: Atlas, 2000. p. 41-50.

PRODANOV, Cleber Cristiano; FREITAS, Ernani Cesar de. Metodologia do trabalho científico: Métodos e Técnicas da Pesquisa e do Trabalho Acadêmico. 2. ed. Novo Hamburgo: Feevale, 2013.

ROSSI, Carlos Alberto Vargas; SLONGO, Luiz Antonio. Pesquisa de Satisfação de Clientes: o Estado-da-Arte e proposição de um método brasileiro. RAC: Revista de Administração contemporânea, Rio de Janeiro, v. 2, n. 1, p.101-125, jan./abr. 1998.

SALOMI, Gilberto Gabriel Eid; MIGUEL, Paulo Augusto Cauchick; ABACKERLI, Alvaro J osé. SERVQUAL x SERVPERF: comparação entre instrumentos para Avaliação da Qualidade de Serviços Internos. Gestão \& Produção, São Paulo, v. 12, n. 2, p. 279-293, maio-ago. 2005.

SILVESTRE, António Luís. Análise de dados e Estatística Descritiva. São Paulo: Escolar, 2007.

STAIR, Ralph M.; REYNOLDS, George W.. Princípios de Sistemas de Informação: uma abordagem gerencial. 6. ed. São Paulo: Pioneira Thomson Learning, 2006.

STREINER, D. L. Being inconsistent about consistency: when coefficient alpha does and doesn't matter. Journal of Personality Assessment, v. 80, p. 217-222, 2003.

URDAN, André Torres; RODRIGUES, Arnaldo Rocha. O Modelo do Índice de Satisfação do Cliente NorteAmericano: um Exame Inicial no Brasil com Equações Estruturais. RAC: Revista de Administração Contemporânea, Rio de Janeiro, v. 3, n. 3, p.109-130, set./dez. 1999. 